\title{
Forensic Entomology/Entomologia Forense Espécies de Oxysarcodexia (Diptera: Sarcophagidae) associadas a carcaças de suínos (Sus scrofa Linnaeus) expostas em um fragmento de Mata Atlântica no município de Salvador, Bahia
}

\author{
Daniele Santos Lopes ${ }^{\bowtie}$, Favízia Freitas de Oliveira1 ${ }^{1}$ Cátia Antunes de Mello-Patiu², \\ Fernanda Maria Pamponet ${ }^{1} \&$ Torriceli Souza Thé $^{3}$
}

1. Universidade Federal da Bahia-Instituto de Biologia-Laboratório de Bionomia, Biogeografia e Sistemática de Insetos. 2. Universidade Federal do Rio de Janeiro - Museu Nacional. 3. Departamento de Polícia Técnica do Estado da Bahia, Laboratório Central da Polícia Técnica, Coordenação de Entomologia Forense.

\section{EntomoBrasilis 11 (2): $103-106$ (2018)}

Resumo. O gênero Oxysarcodexia é encontrado amplamente associado a carcaças de vertebrados em decomposição, sendo, portanto, considerado de grande importância forense. Desta maneira, o objetivo do presente artigo foi conhecer a fauna de Oxysacordexia que visita carcaças de suínos em decomposição em um fragmento de Mata Atlântica no município de Salvador, Bahia. Para isso, quatro carcaças de suínos em decomposição foram utilizados para atração os insetos e expostas no fragmento florestal durante dois períodos: chuvoso (julho a agosto/2012) e seco (novembro a dezembro/2012). Seis espécies de Oxysarcodexia foram atraídas e colonizaram a carcaça: Oxysarcodexia amorosa (Schiner); Oxysarcodexia diana (Lopes); Oxysarcodexia fringidea Curran \& Walley; Oxysarcodexia major Lopes; Oxysarcodexia thornax (Wiedemann); Oxysarcodexia timida (Aldrich). Essas espécies de Oxysarcodexia coletadas mostraram uma aparente preferência pelo período chuvoso e pelas fases finais do processo de decomposição (deterioração avançada e restos).

Palavras-Chave: Carcaças de vertebrados; Decomposição; Entomologia forense; Fauna decompositora; Importância forense.

\section{Species of Oxysarcodexia (Diptera: Sarcophagidae) associated with pig carrion (Sus scrofa Linnaeus) exposed in a fragment of Atlantic Forest in Salvador, Bahia}

\begin{abstract}
The genus Oxysarcodexia is widely found in association with decaying vertebrate carcasses and therefore is considered of great forensic importance. Thus, the aim of this paper was to know the Oxysacordexia fauna visiting rotting pig carcasses in an Atlantic Rain Forest fragment in the Municipality of Salvador, Bahia. Four decomposing pigs carcasses were used to attract insects, exposed in a forest fragment for two seasons: rainy (July-August / 2012) and dry (November to December / 2012). Six species of Oxysarcodexia were attracted and colonized the carcasses: Oxysarcodexia amorosa (Schiner); Oxysarcodexia diana (Lopes); Oxysarcodexia fringidea Curran \& Walley; Oxysarcodexia major Lopes; Oxysarcodexia thornax (Wiedemann); Oxysarcodexia timida (Aldrich). These surveyed Oxysarcodexia species showed an apparent preference for the rainy season and for the final stages of the decomposition process (advanced deterioration and remains).
\end{abstract}

Keywords: Decomposition; Decomposing fauna; Forensic Entomology; Forensic importance; Vertebrate carcasses.

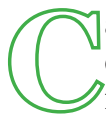
arcaças de vertebrados em decomposição são bons exemplos de recursos efêmeros, com rápidas mudanças nas condições físico-químicas e bióticas, e com altos valores nutritivos. Por isso, são consideradas microambientes ótimos, não só para a ação de fungos e bactérias, mas também para o desenvolvimento de alguns artrópodes, tais como os insetos e, principalmente, os necrófagos, como aqueles das ordens Diptera e Coleoptera (Hansk 1987). Sabe-se também que a velocidade da decomposição pode variar segundo a influência de fatores bióticos, como a atividade dos insetos na carcaça ou de fatores abióticos, tais como temperatura, luminosidade e umidade (NuORTEVA 1974; MARChiori et al. 2000).
Dentre os vertebrados, o uso de carcaça de suínos (Sus scrofa Linnaeus) tem sido o modelo mais aceito nos experimentos em Entomologia Forense para comparação com o ser humano. Tal escolha se deve às similaridades dos porcos domésticos aos humanos, no que se refere ao tegumento com poucos pelos, tamanho da cavidade torácica e características internas (THYSSEN 2000; Tavares 2003; Oliveira-Costa 2007).

Por utilizarem carcaças de suínos em decomposição como fonte de proteínas para o desenvolvimento dos ovários, sítio de cópula e para o desenvolvimento das suas fases imaturas, os dípteros da família Sarcophagidae podem ser utilizados como ferramentas importantes na Entomologia Forense, principalmente para o cálculo da estimativa de Intervalo Pós-Mortem (IPM). Nessa
Edited by:

Roney Rodrigues-Guimarães

Article History:

Received: 29. iii. 2018

Accepted: $14 . v i .2018$
Corresponding author:

Daniele Santos Lopes

\} danys lopes@hotmail.com

(3) http://orcid.org/0000-0001-5702-0086

\section{Funding agencies:}

¿ Fundação de Amparo à Pesquisa do Estado da Bahia - FAPESB; Conselho Nacional de Desenvolvimento Científico e Tecnológico CNPq; Coordenação de Aperfeiçoamento de Pessoal de Nível Superior - CAPES. 
família, são encontrados 23 gêneros de importância forense, dentre eles Oxysarcodexia (CATTS \& GoFf 1992; Souza \& Linhares 1997; Oliveira-Costa 2007).

Oxysarcodexia possui 81 espécies descritas, sendo estas, em sua maioria, coprófagas ou predadoras. No entanto, algumas especies são encontradas amplamente associadas a carcaça de vertebrados em decomposição, tendo sido considerado o gênero com maior diversidade de espécies encontradas associadas a carcaças (Pape 1996; Silva \& Mello-Patiu 2008; Soares \&MelloPatiu 2010; Vasconcelo et al. 2013).

Recentemente, espécimes de Oxysarcodexia riograndense (Lopes) foram encontradas em cadáver humano no Instituto Médico Legal de Recife, confirmando o pressuposto de que as espécies desse gênero se constituem em ferramentas importantes para a Entomologia Forense (OliveIra \& VASCONCElos 2010; VASCONCELos et al. 2014). Desta maneira, o objetivo do presente artigo foi conhecer a fauna de Oxysacordexia que visita corpos em decomposição em um fragmento de Mata Atlântica, através do levantamento das espécies desse gênero visitando carcaças de suínos no Municipio de Salvador, Bahia.

\section{MATERIAL E MÉTODOS}

As coletas foram realizadas em um fragmento de Mata Atlântica dentro do quartel da Polícia do Exército $\left(51^{\circ}\right.$ Centro de Telemáticada Polícia do Exército), localizado em SalvadorBA ( $12^{\circ} 58^{\prime} 27,54$ " S; $38^{\circ} 26^{\prime} 47,68^{\prime \prime}$ W). A área em questão reune formações vegetais diversificadas e heterogêneas, com árvores de grande e médio porte, formando uma mata fechada e densa, reduzindo a penetração de luz em alguns pontos, porém, são detectados também pontos de clareiras em toda a sua extensão (EMBRAPA 1996).

As coletas ocorreram durante o ano de 2012, em dois períodos: período chuvoso (julho-agosto) e período seco (novembrodezembro), sendo utilizadas como isca duas carcaças de suínos de aproximadamente $18 \mathrm{~kg}$, em cada período. Os suínos foram mortos no local do experimento como recomendado pelo Comitê de Ética em Experimentação Animalda Universidade Estadual de Feira de Santana (UEFS), e cobertos com uma gaiola de ferro coberta com uma armadilha tipo Shannon modificada, como proposto em Oliveira-Costa (2007). No topo da armadilha foi deixado um orifício de $10 \mathrm{~cm}$ de diâmetro, onde foi colocado um bocal para o aclopamento de um recipiente transparente (para a entrada de luz), constituindo uma armadilha tipo "PET", que foi trocada todos os dias durante os períodos de coletas. Os espécimes coletados foram levados ao Laboratório de Bionomia, Biogeografia e Sistemática de Insetos (BIOSIS) da Universidade Federal da Bahia, em um recipiente com gelo químico. Para classificar as fases da decomposição da carcaça foi seguida a classificação proposta por BoRNEMISSZA (1957).

Os espécimes foram estudados com o auxílio de microscópio estereoscópico Leica $\mathrm{M} 165 \mathrm{C}$, com uma câmera fotográfica digital Leica DFC295 acoplada e utilizando o Software Leica Application Suite V4.1 Interactive Measurements, Montage. A identificação até o nível de espécie foi realizada com o auxílio de chaves dicotômicas (Carvalho \& Mello-Patiu 2008; Vairo et al. 2011), através de comparações com material da coleção de Sarcophagidae do Museu Nacional, UFRJ).

Em cada período estudado foram computados os dados de temperatura, umidade e pluviosidade, mensurados diariamente pelo Instituto Nacional de Meterologia (INMET).

\section{RESULTADOS E DISCUSSÃO}

Foi coletado um total de 236 espécimes machos de dípteros da família Sarcophagidae, pertencentes ao gênero Oxysarcodexia, distribuídos em seis espécies: Oxysarcodexia amorosa (Schiner);
Oxysarcodexia diana (Lopes); Oxysarcodexia fringidea Curran \& Walley; Oxysarcodexia major Lopes; Oxysarcodexia thornax (Wiedemann) e Oxysarcodexia timida (Aldrich) (Tabela 1).

Embora O. diana, O. thornax e O. timida, já tenham sido registradas em outros estados da Região Nordeste (Lopes \& Tibana 1987; Alves et al. 2014; Barbosa et al. 2015), este é o primeiro registro dessas espécies para a Bahia. No caso de O. major, este estudo também representa seu primeiro registro para a Região Nordeste.

Segundo dados da literatura especializada, as espécies $O$. amorosa, O. diana, $O$ timida e $O$. thornax já haviam sido coletadas em carcaças de suínos em decomposição em trabalhos realizados por Alves (2014) na Paraíba, BARbosa et al. (2009) no Rio de Janeiro, VAiro et al. (2011), no Paraná, Rosa et al. (2011) em Minas Gerais, BARros et al. (2008), no Distrito Federal e Carvalho \& Linhares (2000), em São Paulo. Com relação a $O$. major e $O$. fringidea, esse estudo reporta pela primeira vez sua associação com carcaças de vertebrados em decomposição.

A espécie mais abundante foi $O$. timida, representada por $37,3 \%$ de abundância relativa. Esta espécie foi encontrada tanto no período seco (a partir da fase gasosa), quanto no período chuvoso, (fases finais da decomposição) (Tabela I). Barbosa et al. (2009) também relataram sua presença colonizando carcaças de suínos no Rio de Janeiro.

A espécie $O$. fringidea ocorreu nos dois períodos, porém, com maior abundância no período chuvoso, onde foi encontrada colonizando a carcaça somente nas fases de deterioração avançada e restos. Já no período seco, essa espécie só não colonizou a carcaça na fase fresca.

Observando outros estudos sobre a fauna de Diptera decompositores, $O$. thornax ( $\mathrm{AR}=16 \%$ ) não foi a espécie mais abundante no presente trabalho, o que se contrapõe à maioria dos dados relativos a coletas com carcaças de vertebrados em decomposição, sejam de suíno (BARRos et al. 2008; BARBOSAet al. 2009; VAiro et al. 2011) ou de rato (Beuter et al. 2002; MoretTi et al. 2008). É importante salientar que, adicionalmente, espécimes de $O$. thornax foram também coletadas em cadáver humano (Oliveira-Costa et al .2001), além de que SAlviano (1996) relatou a presença de imaturos dessa espécie se desenvolvendo nas fezes acumuladas no ânus da carcaça em decomposição, demonstrando que a mesma pode ser, portanto, considerada como de grande importância forense.

Do ponto de vista do processo de decomposição, as cinco fases propostas por Bornemissza (1957) foram identificadas durante os períodos de coleta em Salvador, e caracterizadas da seguinte forma: fresca - ausência de odor e de sinais externos de decomposição, porém, nela já foram observados presença de alguns dípteros; gasosa - inchamento da carcaça, com a ocorrência de membros rigidos e um leve odor de putrefação; deterioração inicial - colapso do corpo, e exposição dos orgãos internos, com odor de putrefação intenso; deterioração avançada algumas partes da carcaça encontravam-se secas e com fungos, apresentando os ossos parcialmente expostos e com odor de putrefação leve; restos - exposição total dos ossos, com odor extremamente suave.

No presente experimento, as coletas foram realizadas diariamente, com acompanhamento da carcaça até $05^{\circ}$ dia de decomposição, nos dois períodos, com os dados coletados até mesmo quando a carcaça se encontrava com os ossos totalmente expostos e secos, quando foi observada uma grande quantidade de insetos da ordem Coleoptera e quase nenhum inseto da ordem Diptera.

Quanto ao tempo de decomposição, no período chuvoso a carcaça demorou 11 dias para alcançar a fase de restos, enquanto que no 
período seco esse mesmo processo durou apenas 8 dias. Nesse sentindo, Bornemissza (1957) e MARCHIORI (2000) discutem que a velocidade da decomposição pode realmente variar segundo a ação de fatores abióticos, especialmente a temperatura, umidade, precipitação e luz.

Relacionando os dados de abundância nos dois períodos amostrados, notou-se uma aparente preferência dos indivíduos do gênero Oxysarcodexia pelo período chuvoso, com 138 espécimes coletadas neste período $(58,4 \%$ dos indivíduos coletados em todo o período de experimento). Notou-se também maior abundância e diversidade de espécie nas fases finais de decomposição (deterioração avançada e restos), não tendo sido coletado nenhum espécime na fase fresca.

Com relação a esse aspecto, existem controvérsias entre os autores quanto à "preferência" das espécies de Sarcophagidae pelas fases da decomposição, sendo que CARvalHo \& Linhares (2000) relataram maior abundância nas fases finais, enquanto que BARros et al. (2008) citaram que a maior abundância ocorreu na fase gasosa. Especificamente para representantes do gênero Oxysarcodexia, segundo Barros et al. (2008) e Rosa et al. (2011), as espécies podem ocorrer em todas as fases, porém, poucas espécies são encontradas colonizando a fase fresca.

No caso das fêmeas da família Sarcophagidae de uma forma geral, dados da literatura sugerem que o fato de serem ovovivíparas, pondo larvas de $1^{\mathrm{O}}$ instar, que se alimentam imediatamente da carcaça (ao contrário de Calliphoridae, por exemplo, que oviposita e, por isso, os imaturos necessitariam de um tempo até a eclosão da larva 1) (DENNo \& CoTHRAN 1976), levaria a uma tendência das fêmeas de Sarcophagidae a procurarem carcaças a partir da segunda fase da decomposição, principalmente, quando os recursos alimentares estariam mais facilmente disponíveis para a alimentação das larvas (ferimentos, orifícios naturais e fezes mais expostos).

Neste sentido, o presente trabalho fornece uma contribuição importante para o avanço da Entomologia Forense na Região Nordeste, uma vez que a lista de espécies do gênero Oxysarcodexia de importância forense e suas associações com cada fase da decomposição apresentadas aqui, funcionará como ferramenta importante, possibilitando que os dados obtidos sobre esses insetos possam ser utilizados pelo Departamento de Polícia Técnica da Bahia na elucidação de crimes.

Tabela 1. Espécies de Oxysarcodexia coletadas durante o período seco e chuvoso nas fases de decomposição. (I) Fase fresca; (II) Gasosa; (III) Deterioração inicial; (IV) Deterioração avançada; (IV) Restos. * AA - Abundância absoluta *AR - Abundância relativa

\begin{tabular}{|c|c|c|c|c|c|c|c|c|c|c|c|c|c|c|}
\hline \multirow{2}{*}{ Espécies } & \multicolumn{6}{|c|}{ Período chuvoso } & \multicolumn{6}{|c|}{ Período seco } & \multirow{2}{*}{$\mathbf{A A} *$} & \multirow{2}{*}{ AR \%* } \\
\hline & $\mathbf{I}$ & II & III & IV & $\mathbf{V}$ & Total & $\mathbf{I}$ & II & III & IV & $\mathbf{V}$ & Total & & \\
\hline Oxysarcodexia amorosa (Schiner) & o & o & o & 3 & 4 & 7 & o & 18 & o & 14 & 6 & 38 & 45 & $19 \%$ \\
\hline Oxysarcodexia diana (Lopes) & o & o & o & 1 & 1 & 2 & o & 3 & 1 & 1 & 2 & 7 & 9 & $4 \%$ \\
\hline Oxysarcodexia fringidea Curran \& Walley & o & o & o & 4 & 41 & 45 & o & 4 & 1 & 2 & 1 & 8 & 53 & $22.4 \%$ \\
\hline Oxysarcodexia major Lopes & o & 2 & o & o & 1 & 3 & o & o & o & o & o & o & 3 & $1.3 \%$ \\
\hline Oxysarcodexia thornax (Wiedemann) & o & o & o & 7 & 20 & 27 & o & 4 & o & 6 & 1 & 11 & 38 & $16 \%$ \\
\hline Oxysarcodexia timida (Aldrich) & 0 & 0 & o & 36 & 18 & 54 & 0 & 14 & 2 & 15 & 3 & 34 & 88 & $37.3 \%$ \\
\hline Total & o & 2 & o & 51 & 85 & 138 & o & 43 & 4 & 39 & 13 & 98 & 236 & $100 \%$ \\
\hline
\end{tabular}

\section{AGRADECIMENTOS}

À Fundação de Amparo à Pesquisa do Estado da Bahia (FAPESB) pelo financiamento do projeto. À Universidade Federal da Bahia (UFBA) pelo apoio logístico. À equipe do Laboratório de Entomologia Forense do Departamento de Polícia Técnica da Bahia e do Laboratório de Bionomia, Biogeografia e Sistemática de Insetos (BIOSIS-UFBA) pelo auxilio nas coletas, montagem e identificação do material. Ao Conselho Nacional de Desenvolvimento Científico e Tecnológico - CNPq pela bolsa de pesquisa a CAMP (Proc. 309170/2015-0) e a Coordenação de Aperfeiçoamento de Pessoal de Nível Superior - CAPES pela bolsa de mestrado.

\section{REFERÊNCIAS}

Alves, A.C.F., W.E. dos Santos \& A.J. Creão-Duarte, 2014. Diptera (Insecta) de importância forense da região Neotropical. Entomotropica, 29: 77-94.

Barbosa, R.R., C.A. Mello-Patiu, R.P. Mello \& M.M.C. Queiroz, 2009. New records of calyptrate (Faniidae, Muscidae and Sarcophagidae) associated with the decomposition of domestic pigs in Brazil. Memórias Instituto Oswaldo Cruz, 104: 923-926. DOI: https://doi.org/10.1590/s007402762009000600018.

Barros, R.M., C.A. Mello-Patiu \& J.R. Pujol-Luz, 2008. Sarcophagidae (Insecta, Diptera) associados à decomposição de carcaças de Sus scrofa Linnaeus (Suidae) em área de Cerrado do Distrito Federal, Brasil. Revista Brasileira de Entomologia, 52: 606-609. DOI: https://doi.org/10.159o/ s0085-56262008000400011.
Beuter, L., P.A. Fernandes, P.B. Barros, C.R. Souza \& J. Mendes, 2012. Insetos de potencial importância forense e na saúde pública em região urbana de Minas Gerais: frequência relativa e variação sazonal de fauna atraída e criada em carcaças de roedores. Revista de Patologia Tropical, 41: 480-490. DOI: https://doi.org/10.5216/rpt.v41i4.21711.

Bornemissza, G.F., 1957. Analysis of arthropod succession in carrion and the effect of its decomposition on the soil fauna. Australian Journal of Zoology, 5: 1-12. DOI: https://doi.org/10.1071/zo9570001.

Carvalho, J. B. \& C. A Mello-Patiu. 2008. Key to the adults of the most common forensic species of Diptera in South America. Revista Brasileira de Entomologia, 52: 390-406. DOI: https://doi.org/10.1590/s0085-56262008000300012.

Carvalho, L.M.L. \& A.X. Linhares, 2001. Seasonality of insect succession and pig carcass decomposition in a natural forest area in southeastern Brazil. Journal of Forensic Sciences, 46: 604-608. DOI: https://doi.org/10.1520/ifs15011j.

Catts, E.P. \& M.L. Goff, 1992. Forensic entomology in criminal investigations. Annual Review of Entomology, 27: 253-272. DOI: https://doi.org/10.1146/annurev.ento.37.1.253.

Denno, R.F. \& W.R. Cothran, 1976. Competitive interactions and ecological strategies of sarcophagid and calliphorid flies inhabiting rabbit carrion. Annals of the Entomological Society of America, 69:109-13. DOI: https://doi.org/10.1093/ aesa/69.1.109.

Embrapa - Empresa Brasileira de Agropecuária, 1996. Atlas do meio ambiente do Brasil. $2^{\mathrm{a}}$ edição, Editora Terra Viva, Brasília (DF), 16op.

Hanski, I., 1986. Nutritional ecology of dung and carrion feeding insects, p. 837-884. In: Slansky F \& Rodriguez JG. (Ed.). 
Nutrional ecology of insects, mites and spiders. Jonh Wiley. $1016 \mathrm{p}$.

Marchiori, C.H., C.G. Silva, E.R. Caldas, C.I.S. Vieira, K.G.S. Almeida, F.F. Teixeira \& A.X. Linhares, 2000. Artrópodes associados com carcaça de suíno em Itumbiara, sul de Goiás. Arquivos do Instituo de Biologia, 67: 167-170.

Moretti, T.C., O.B. Ribeiro, P.J. Thyssen \& D.R. Solis, 2008. Insects on decomposing carcasses of small rodents in a secondary Forest in Southeastern Brazil. European Journal of Entomology, 105: 691-696. DOI: https://doi.org/10.14411/ eje.2008.094.

Nuorteva, P., 1974. Age determination of blood stain in a decaying shirt by entomological means. Journal of Forensic Sciences, 3: 89-94. DOI: https://doi.org/10.1590/s010209352000000500009.

Oliveira, T.C. \& S.D. Vasconcelos, 2010. Insects (Diptera) associated with cadavers at the Institute of Legal Medicine in Pernambuco, Brazil: Implications for forensic entomology. Forensic Science International, 198: 97-102. DOI: https://doi.org/10.1016/j.forsciint.2010.01.011.

Oliveira-Costa, J., 2007. Entomologia forense: quando os insetos são vestígios. São Paulo: Editora Millenium, $2^{\circ}$ edição, 456 p.

Pape, T., 1996. Catalogue of Sarcophagidae of the World (Insecta, Diptera). vol. 8. Stockholm: Memoirs on Entomology, International. $558 \mathrm{p}$.

Rosa, T.A., M.L.Y. Babata, C.M. Souza, D. Sousa, C.A. MelloPatiu, F.Z. Vaz-De-Mello \& J. Mendes, 2011. Arthropods associated with pig a carrion in two vegetation profiles of Cerrado in the State of Minas Gerais, Brazil. Revista Brasileira de Entomologia, 55: 424-434. DOI: https://doi.org/10.1590/ s0085-56262011005000045.

Salviano, R.J.B., 1996. Sucessão de Diptera Caliptrata em carcaça de Sus scrofa Linnaeus, Rio de Janeiro, RJ, Brasil. Dissertação (Mestrado). Universidade Federal Rural do Rio de Janeiro, $158 \mathrm{f}$.

Silva, K.P. \& C.A. Mello-Patiu, 2008. Morfologia comparada da terminália masculina de quatro espécies de Oxysarcodexia Townsend, 1917 (Diptera, Sarcophagidae). Arquivos do Museu Nacional, 66: 363-372.
Soares, W.F. \& C.A. Mello-Patiu, 2010. Two new Neotropical species of the genus Oxysarcodexia Townsend (Diptera, Sarcophagidae). Revista Brasileira de Entomologia, 54: 72-75. DOI: https://doi.org/10.1590/s0085-56262010000100008.

Souza, A.M. \& A.X. Linhares, 1997. Diptera and Coleoptera of potential forensic importance in Southeastern Brazil: relative abundance and seasonality. Medical and Veterinary Entomology, 11: 8-12. DOI https://doi.org/10.1111/j.13652915.1997.tboo284.x.

Tavares, M.C., 2003. Sucessão faunística de populações de insetos associados à decomposição de carcaças de suínos expostas em diferentes altitudes e condições pluviométricas na reserva florestal da Serra do Japi, Jundiaí, SP. Tese (Doutorado). São Paulo: Universidade Estadual de Campinas. 120 f. Handle: http://repositorio.unicamp.br/jspui/handle/ REPOSIP/314292.

Thyssen, P.J., 2000. Decomposição e sucessão entomológica em carcaças de suínos (Sus scrofa L.) de tamanhos diferentes: estudos em ambiente de mata natural na região de Campinas - SP. Campinas, SP. Dissertação (Mestrado). São Paulo: Universidade Estadual de Campinas. $85 \mathrm{f}$.

Vairo, K.P., C.A. Mello-Patiu \& C.J.B. Carvalho, 2011. Pictorial identification key for species of Sarcophagidae (Diptera) of potential forensic importance in southern Brazil. Revista Brasileira de Entomologia, 55: 333-347. DOI: https://doi.org/10.1590/s0085-56262011005000033.

Vasconcelos, D.S., T.F. Soares \& D.L. Costa, 2014. Multiple colonization of a cadaver by insects in an indoor environment: first record of Fannia trimaculata (Diptera: Fanniidae) and Peckia (Peckia) chrysostoma (Sarcophagidae) as colonizers of a human corpse. International Journal of Legal Medicine 128: 229. DOI: https://doi.org/10.1007/s00414-013-0936-2.

Vasconcelos, S.D., T.M. Cruz, R.L. Salgado \& P.J. Thyssen, 2013. Dipterans associated with a decomposing animal carcass in a rainforest fragment in Brazil: notes on the early arrival and colonizationbynecrophagousspecies.Journal of InsectScience 13: 1-11. DOI: https://doi.org/10.1673/031.013.14501.

\section{Suggestion citation:}

Lopes, D.S., F.F. Oliveira, C.A. Mello-Patiu, F.M. Pamponet \& T.S. Thé, 2018. Espécies de Oxysarcodexia (Diptera: Sarcophagidae) associadas a carcaças de suínos (Sus scrofa Linnaeus) expostas em um fragmento de Mata Atlântica no município de Salvador, Bahia. EntomoBrasilis, 11 (2): 103-106.

Available on: doi:10.12741/ebrasilis.v11i2.779
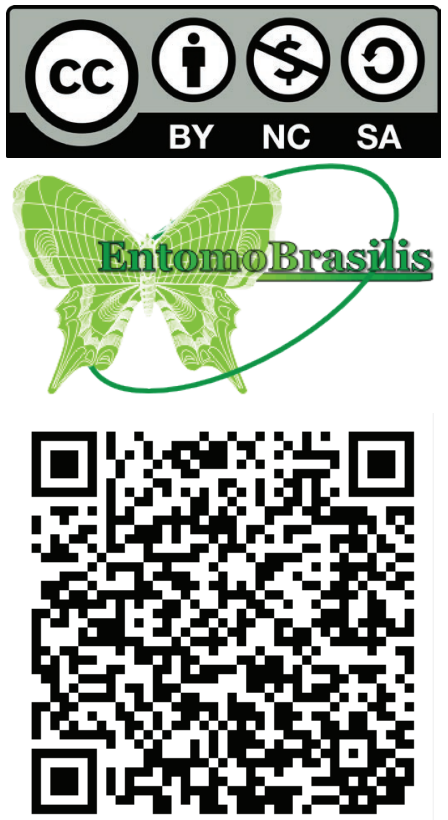